EPJ manuscript No.

(will be inserted by the editor)

\title{
Active Brownian motion in a narrow channel
}

\author{
Xue Ao ${ }^{1}$, Pulak K. Ghosh ${ }^{2}$, Yunyun Li $^{3, a}$, Gerhard Schmid ${ }^{1}$, Peter Hänggi ${ }^{1,3}$ and \\ Fabio Marchesoni ${ }^{3,4}$ \\ 1 Institut für Physik, Universität Augsburg, D-86135 Augsburg, Germany \\ 2 Department of Chemistry, Presidency University, Kolkata - 700073, India \\ 3 Center for Phononics and Thermal Energy Science, School of Physic Science and Engi- \\ neering, Tongji University, Shanghai 200092, People's Republic of China \\ ${ }^{4}$ Dipartimento di Fisica, Università di Camerino, I-62032 Camerino, Italy
}

\begin{abstract}
We review recent advances in rectification control of artificial microswimmers, also known as Janus particles, diffusing along narrow, periodically corrugated channels. The swimmer self-propulsion mechanism is modeled so as to incorporate a nonzero torque (propulsion chirality). We first summarize the effects of chirality on the autonomous current of microswimmers freely diffusing in channels of different geometries. In particular, left-right and upside-down asymmetric channels are shown to exhibit different transport properties. We then report new results on the dependence of the diffusivity of chiral microswimmers on the channel geometry and their own self-propulsion mechanism. The self-propulsion torque turns out to play a key role as a transport control parameter.
\end{abstract}

\section{Introduction}

Rectification of Brownian motion in a narrow, periodically corrugated channel has been the focus of a concerted effort [1/2 3] aimed at establishing net particle transport in the absence of external biases. To this purpose two basic ingredients are required: a spatial asymmetry of the channel and a time correlation of the perturbations, random or deterministic, applied to the diffusing particles. The ensuing spontaneous current is a manifestation of the so-called ratchet effect 2]. Typically, demonstrations of the ratchet effect had recourse to external unbiased time-dependent drives (rocked and pulsated ratchets). Rectification induced by time-correlated, or colored, non-equilibrium fluctuations (thermal ratchets) is conceptually feasible, but has been, so far, of limited practical use. The idea in itself, however, is appealing: The diffusing particles would harvest kinetic energy directly from their non-equilibrium environment, without requiring any externally applied field at all, and transport would ensue as an autonomous symmetry-directed particle flow.

To enhance rectification of time correlated diffusion in a modulated channel with zero drives, we recently proposed 4 to make use of a special type of diffusive tracers, namely of active, or self-propelled, Brownian particles. Self-propulsion is the ability of most living organisms to move, in the absence of external drives, thanks to an "engine"

\footnotetext{
a e-mail: yunyunli@tongji.edu.cn
} 

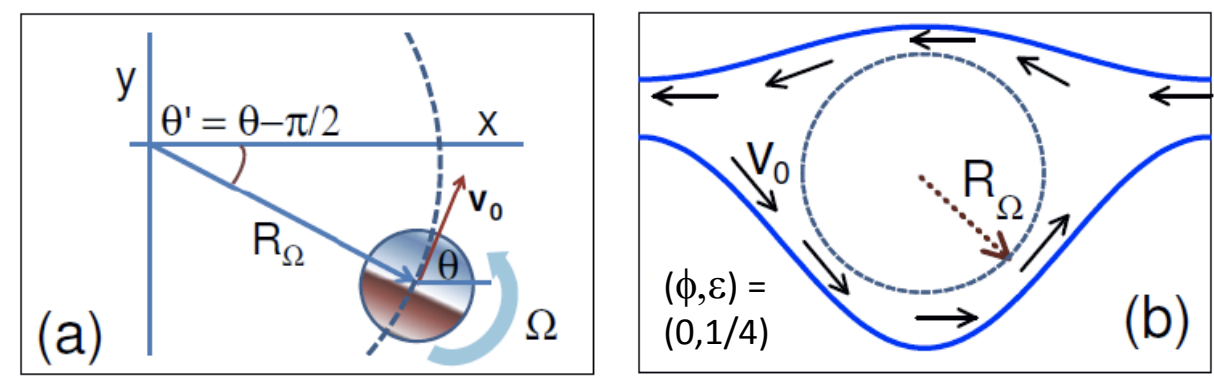

Fig. 1. (Color online) Levogyre Janus particle in a narrow channel: (a) noiseless particle with velocity $\mathbf{v}_{0}$ and torque frequency $\Omega>0$, Eq. (1), moving along a circular arc of radius $R_{\Omega}$ (dashed line); (b) upside-down asymmetric channel compartment, Eq. (3) with $\phi=0$ and $\epsilon=0.25$. In (b) the open boundary-trajectories and a closed circular trajectory of radius $R_{\Omega}$ are drawn for explanatory purposes (see Sec. 3.2 for actual simulation data).

of their own [5. Optimizing self-propulsion of micro- and nano-particles (artificial microswimmers) is a growing topic of today's nanotechnology [6/7/8. Recently, a new type of artificial microswimmers has been synthesized [9]10, where self-propulsion takes advantage of the local gradients asymmetric particles can generate in the presence of an external energy source (self-phoretic effects). Such particles, called Janus particles (JP), consist of two distinct "faces", only one of which is chemically or physically active. Thanks to their functional asymmetry, these active particles can induce either concentration gradients (self-diffusiophoresis) by catalyzing a chemical reaction on their active surface 11/1213, or thermal gradients (self-thermophoresis), e.g., by inhomogeneous light absorption [14] or magnetic excitation [15.

A self-propulsion mechanism acts on an pointlike particle by means of a force and, possibly, a torque. In the absence of a torque, the line of motion is directed parallel to the self-phoretic force and the JP propels itself along a straight line, until it changes direction, due to gradient fluctuations [16 or random collisions against other particles or geometric boundaries [17. This is the highly stylized case mostly studied in the recent literature, where, for simplicity, the JPs are assumed to be rotationally symmetric around their line of motion (symmetric JPs). In the presence of an additional torque the self-phoretic force and the line of motion are no longer aligned and the microswimmer tends to execute circular orbits [18]19.

Active chiral motion has long been known in biology [20|19|21] and more recently observed in asymmetrically propelled micro- and nano-rods: A torque can be intrinsic to the propulsion mechanism, due to the presence of geometrical asymmetries in the particle fabrication, engineered or accidental (asymmetric JP's) 22|24|23, or externally applied, for instance, by laser irradiation 14 or hydrodynamic fields 25 . In the finite damping regime, the Lorentz force exerted by a magnetic field on a charged active Brownian particle also amounts to an external torque [26; however, the effects of such magnetic torques vanishes in the overdamped limit [27].

In this paper we discuss the interplay of chiral propulsion and channel spatial asymmetry on controlling autonomous rectification. In Sec. 2 we remind that active Brownian motion is time correlated per se, which means that transport control of, say, a JP with assigned self-propulsion properties, can only be achieved by suitably tailoring the channel boundaries. In Sec. 3 we briefly review autonomous rectification of JP's with and without external torque. In both cases the net on-plane drive exerted on the particle is null (unbiased diffusion). We distinguish between two categories of channels, left-right asymmetric channels, Sec. 3.1. where even nonchiral JP's can be rectified, and upside-down asymmetric channels, Sec. 3.2. where particle ratcheting 


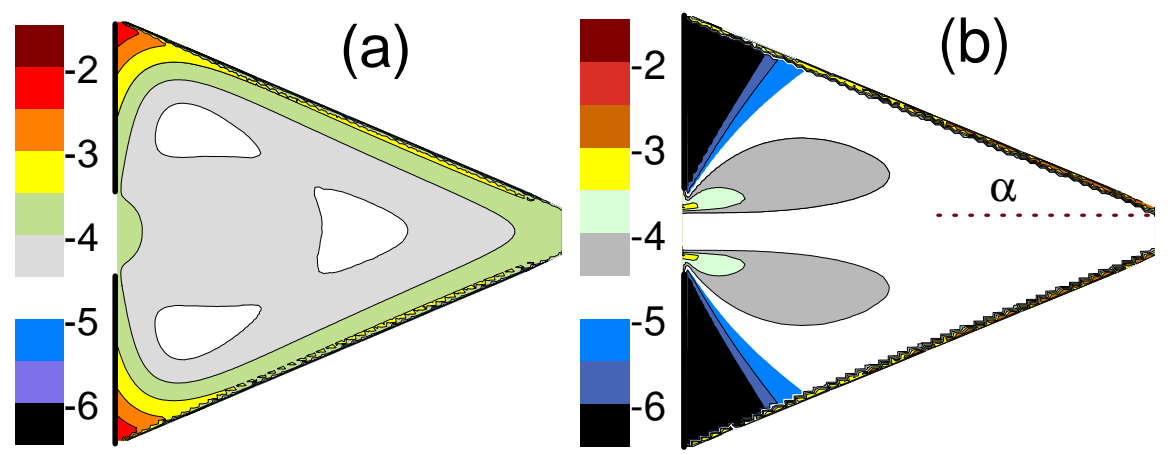

Fig. 2. (Color online) Logarithmic contour plots of the stationary particle density, $P(x, y)$, in a triangular compartment of size $x_{L}=y_{L}=1$ and pore width $\Delta=0.1$; the compartment acts as a funnel of angular width $2 \alpha$. Other simulation parameters are: $D_{\theta}=0.006, v_{0}=1$ and (a) $D_{0}=0.03$, (b) $D_{0}=0$ (no thermal noise); sliding b.c. have been adopted throughout. Both densities are singular at the corners and along the side walls, where graphics resolution effects are apparent.

requires a nonzero torque. Based on numerical evidence, in Sec. 3.3 we establish the minimal asymmetry conditions that make rectification of active Brownian particles possible. Finally, in Sec. 4 we present new results on active diffusion in channels of various geometries. In particular, we show that diffusion of JP's is not controlled by the channel geometry as much as by the angular asymmetry of the self-propulsion mechanim. The diffusion of nonchiral and chiral JP's is investigated in Secs. 4.1 and 4.2. respectively. Ideas for future work are discussed in the concluding Sec. 5.

\section{Model}

In order to avoid unessential complications, we restrict this report to the case of $2 \mathrm{D}$ channels and pointlike artificial microswimmers of the JP type [9]. A chiral JP gets a continuous push from the suspension fluid, which in the overdamped regime amounts to a rotating self-propulsion velocity $\mathbf{v}_{0}$ with constant modulus $v_{0}$ and angular velocity $\Omega$. Additionally, the self-propulsion direction varies randomly with time constant $\tau_{\theta}$, under the combined action of thermal noise and orientational fluctuations intrinsic to the self-propulsion mechanism. Accordingly, the microswimmer mean free selfpropulsion path approximates a circular arc of radius $R_{\Omega}=v_{0} /|\Omega|$ and length $l_{\theta}=$ $v_{0} \tau_{\theta}$ [18. Chiral effects are prominent when $R_{\Omega} \lesssim l_{\theta}$, or equivalently, $|\Omega| \tau_{\theta} \gtrsim 1$ (strong chirality regime).

The bulk dynamics of such an overdamped chiral JP obeys the Langevin equations (LE) 18

$$
\begin{aligned}
& \dot{x}=v_{0} \cos \theta+\xi_{x}(t) \\
& \dot{y}=v_{0} \sin \theta+\xi_{y}(t) \\
& \dot{\theta}=\Omega+\xi_{\theta}(t),
\end{aligned}
$$

where $\mathbf{r}=(x, y)$ are the coordinates of a particle subject to the Gaussian noises $\xi_{i}(t)$, with $\left\langle\xi_{i}(t)\right\rangle=0$ and $\left\langle\xi_{i}(t) \xi_{j}(0)\right\rangle=2 D_{0} \delta_{i j} \delta(t)$ for $i=x, y$, modeling the equilibrium thermal fluctuations in the suspension fluid. The channel is directed along the $x$ axis, the self-propulsion velocity is oriented at an angle $\theta$ with respect to it and the sign of $\Omega$ is chosen so as to coincide respectively with the positive (levogyre) and negative 
(dextrogyre) chirality of the swimmer, see Fig. 1(a). The orientational fluctuations of the propulsion velocity are modeled by the Gaussian noise $\xi_{\theta}(t)$ with $\left\langle\xi_{\theta}(t)\right\rangle=0$ and $\left\langle\xi_{\theta}(t) \xi_{\theta}(0)\right\rangle=2 D_{\theta} \delta(t)$, where the noise strength $D_{\theta}$ is the relaxation rate of the self-propulsion velocity, $D_{\theta}=2 / \tau_{\theta}$, see Sec. 4.1 for more details.

In this work the JP's were assumed to be pointlike as we intended to focus on the causes of autonomous transport and its control. However, numerical and experimental evidence clearly show that the dynamical parameters of a real JP in the bulk, i.e., its self-propulsion speed, friction coefficient, thermal and rotational diffusion coefficients 28 and effective shape, all depend on its size as well as on its shape [29. These effects can be accounted for, at least qualitatively, by an appropriate choice of the free parameters introduced in our model Eqs. (1).

The simplifications introduced here are not limited to the dimensionality of the channel or the size of the particle. All noise sources in Eq. (1) have been treated as independently tunable, although, strictly speaking, thermal and orientational fluctuations may be statistically correlated (see, e.g., 21]). Moreover, we ignored hydrodynamic effects, which not only favor clustering in dense mixtures of JP's [30]31, but may even cause their capture by the channel walls 32 . However, we made sure that the parameters used in our simulations are experimentally accessible, as apparent on expressing times in seconds and lengths in microns (see Refs [1321] for a comparison).

When confined to a channel directed along the $x$ axis, the particle transverse coordinate, $y$, is bounded by the wall functions $w_{ \pm}(x), w_{-}(x) \leq y \leq w_{+}(x)$. All wall geometries considered below are periodic with compartment length $x_{L}$, namely $w_{ \pm}\left(x+x_{L}\right)=w_{ \pm}(x)$. The channel compartments are connected by pores of width $\Delta$, much narrower than their maximum cross-section.

Simulating a constrained JP requires defining its collisional dynamics at the boundaries. For the translational velocity $\dot{\mathbf{r}}$ we assumed elastic reflection. Regarding the coordinate $\theta$ we considered two possibilities:

(a) frictionless collisions, $\theta$ unchanged. The active particle slides along the walls for an average time of the order of $\tau_{\theta}$, until the $\theta(t)$ fluctuations redirect it toward the interior of the compartment. For simplicity, all simulation results presented in this report have been obtained for sliding b.c. The panels of Fig. 2 show how the stationary particle probability density $P(x, y)$ accumulates along the boundaries; this effect is even stronger in the noiseless case, $D_{0}=0$;

(b) rotation induced by a tangential friction, $\theta$ randomized. These b.c. cause the particle to diffuse away from the boundary, which, as discussed below, tends to weaken the rectification effect. Note that, as pointed out in Refs. 33, should one assume elastic boundary reflection for both $\dot{\mathbf{r}}$ and $\mathbf{v}_{0}$, then the self-propelled motion of a JP would coincide with an ordinary Brownian motion with finite damping constant, $\gamma=2 / \tau_{\theta}$, and self-diffusion constant $D_{s}$ defined in Sec. 4.1. Being that an equilibrium random process, it could not be rectified, no matter what the spacial asymmetry of the channel.

We further stress that on modeling the boundary conditions we heavily relied on the pointlike particle assumption to neglect (i) the dependence of the self-propulsion mechanism on the particle distance from the walls; (ii) the hydrodynamic interactions between particle and walls [29]; and (iii) the ensuing particle accumulation against the walls 32 .

Finally, Eqs. (1) have been numerically integrated by using a standard Milstein algorithm [4] with a very short time step, $10^{-5}-10^{-7}$, to ensure numerical stability. As initial conditions we have assumed that at $t=0$ the particle is uniformly distributed with random orientation in a channel compartment located between $x=0$ to $x=x_{L}$. The total observation time was set to $10^{4} \times \tau_{\theta}$, or $10^{4} \times \Omega^{-1}$, or $10^{4}$, whichever is greater, so that effects due to the initial conditions and transient processes can 
be neglected. The results reported in the figure shown here have been obtained by ensemble averaging over $10^{4}-10^{6}$ trajectories, depending on the observable.

\section{Autonomous currents}

We discuss first autonomous rectification of a JP in two different classes of $2 \mathrm{D}$ asymmetric channels. To characterize its drift in the absence of external biases, we introduce the rectification power

$$
\eta=|\bar{v}| / v_{0},
$$

where the net drift velocity of the particle, $\bar{v}=\lim _{t \rightarrow \infty}\langle x(t)-x(0)\rangle / t$, is expressed in units of its self-propulsion velocity, $v_{0}$.

\subsection{Left-right asymmetric channels}

The LE system of Eq. (1) was first numerically simulated in for a JP confined to a directed channel made of triangular compartments with dimensions $x_{L} \times y_{L}$ and pore size $\Delta$, see Fig. 2. The compartment aspect ratio was kept constant, $r=x_{L} / y_{L}=1$, and by rescaling the coordinates $x$ and $y$ by an appropriate factor $\kappa, x \rightarrow x / \kappa$ and $y \rightarrow y / \kappa$, its dimensions can always be conveniently rescaled to $x_{L}=y_{L}=1$ (as done in Figs. 2 5 and 11). Analogously, by time rescaling, $t \rightarrow v_{0} t / \kappa$, one can work with self-propulsion velocities of constant modulus, $v_{0}=1$. In conclusion, the output of our numerical analysis only depends on four characteristic lengths: the pore width, $\Delta$; the thermal length, $D_{0} / v_{0}$; the self-propulsion length, $l_{\theta}$; and the chiral radius, $R_{\Omega}$, all to be compared with the compartment dimensions set to one. Throughout our simulation work we assumed narrow pores and low thermal noise, so that the first two lengths play no key role in the discussion of our results [see Eq. (5)]. Equivalently, when appropriate, instead of the ratios $l_{\theta} / x_{L}$ and $R_{\Omega} / x_{L}$ one can make use of the dimensionless quantities $\tau_{\theta} / \tau_{x}$ and $|\Omega| \tau_{\theta}$, where $\tau_{\theta}$ is the selfpropulsion time, $\tau_{x}=x_{L} / v_{0}$ a characteristic compartment crossing time, and $\Omega$ the chiral angular frequency.

Note that, in view of their orientation, the triangular compartments of Fig. 2 tends to funnel the particle to the right (easy-flow direction) with $\bar{v}>0$. The magnitude of this effect depends on the modulus and not on the sign of $\Omega$.
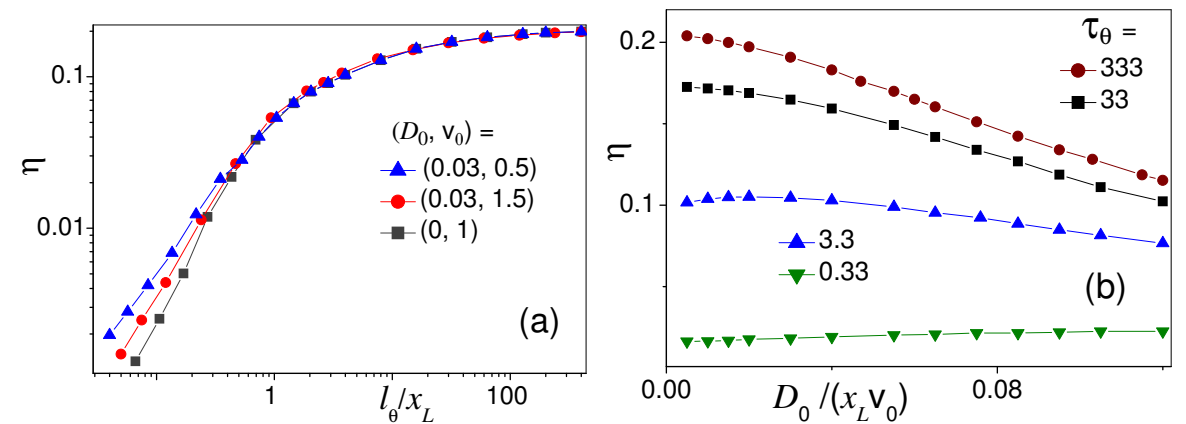

Fig. 3. (Color online) Rectification of a nonchiral JP in a triangular channel with compartment geometry as in Fig. 2 (a) rectification power, $\eta$ (solid symbols) vs $l_{\theta}=v_{0} \tau_{\theta}$ for different $D_{0}$ and $v_{0}$; (b) $\eta$ vs. $\bar{D}_{0}$ for $v_{0}=1$ and different $\tau_{\theta}$. The particle flow is oriented to the right, i.e., $\bar{v}>0$. 
Non-chiral Janus particles, $\Omega=0$. This is the case first reported in Ref. [4]. When the Janus self-propulsion length $l_{\theta}$ is larger than the compartment dimensions, the particle undergoes several collision, during the time interval $\tau_{\theta}$ (Knudsen regime 34 ). As the Janus dynamics gets more sensitive to the compartment asymmetry, the curves $\bar{v}\left(\tau_{\theta}\right)$ increase monotonously with $\tau_{\theta}$, until they level off to an asymptotic upper bound [35], see Fig. 3(a). Most importantly, such asymptotic $\eta$ values are much larger than the rectification power of the thermal ratchets investigated in the earlier literature 2 .

The impact of thermal noise on the rectification of a JP can be summarized as follows. In Ref. [4] we showed that for $l_{\theta} \ll x_{L}$ the self-propulsion velocity changes orientation before the particle slides along a compartment side and through the pore. Thermal noise, by pulling the particle towards the central lane of the channel, acts as a lubricant. On the contrary, for $l_{\theta} \gg x_{L}$, thermal fluctuations help the particle overcome the blocking action of the compartment corners, see Fig. 2, thus suppressing rectification. In the intermediate regime, where $\eta$ is the strongest, these two opposite actions of thermal noise coexist, as illustrated in Fig. 3(b), thus defining an optimal thermal noise level.

Finally, in view of practical applications, we tested the robustness of JP rectification in channels with variable degrees of asymmetry: (i) In Fig. 4 we modified the compartment geometry by shifting the corner coordinate, $x_{0}$, in the range $\left[0, x_{L} / 2\right)$ (see inset). One immediately sees that $\eta$ decreases by only a factor 2 for $x_{0}$ up to 0.2 ; (ii) In our previous report 4 we studied the consequence of rescaling the $x$ and $y$ compartment dimensions by a factor $\kappa$. We concluded that rectification is rather insensitive to $\kappa$ in the Knudsen regime, $l_{\theta}>\kappa x_{L}$. For exceedingly large $\kappa$, the intensity of the rescaled translational (thermal) noise, $D_{0} / \kappa v_{0}$, is suppressed with respect to the intensity of the rescaled propulsion noise, $\kappa D_{\theta} / v_{0}$, which means that the role of thermal fluctuation grows negligible on increasing $\kappa$. As a consequence, in this limit $\eta$ approaches a constant, that is, $\bar{v}$ is inverse proportional to $\kappa$; (iii) In Ref. [4], we also reported that for narrow pores, $\Delta \ll y_{L}, \eta$ slightly decreases with lowering $\Delta$. The explanation is simple. As the pore shrinks, the compartment sidewalls grow longer and the particle takes more time to slide along them up to the exit pore. On the other hand, the negative flow is blocked mostly at the compartment corners, regardless the actual pore size. This result indicates that our numerical analysis can be

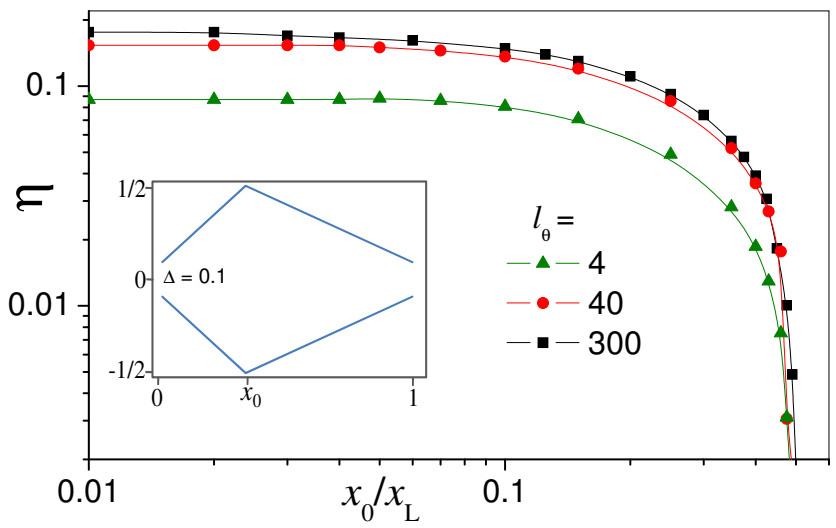

Fig. 4. (Color online) Rectification of nonchiral JP with $v_{0}=1$ in asymmetric channels with different geometries. A typical compartment is sketched in the inset: $x_{L}, y_{L}$, and $\Delta$ are as in Fig. 2, but the corners are shifted by $x_{0}$. Rectification power $\eta$ vs. $x_{0}$ for $D_{0}=0.03$, and different $D_{\theta}=2 v_{0} / l_{\theta}$. The particle orientation is as in Fig. 3 for all $x_{0}=0$. 

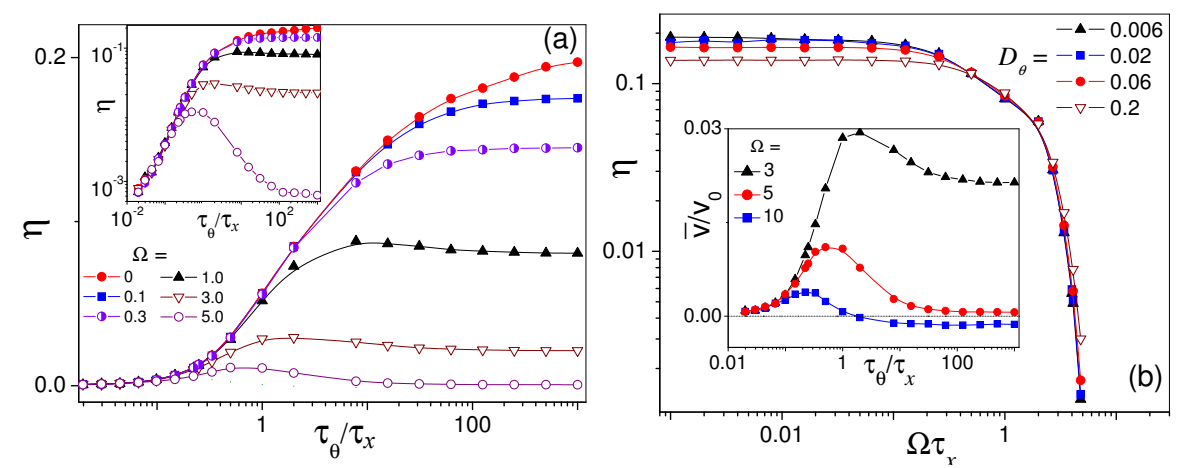

Fig. 5. (Color online) Rectification of a chiral JP in a triangular channel: (a) $\eta$ vs. $\tau_{\theta}$ for different $\Omega$; the same data sets are plotted in a semi-logarithmic (main panel) and bilogarithmic graph (inset); (b) $\eta$ vs. $\Omega$ for different $\tau_{\theta}$. Here, $\tau_{x} \equiv x_{L} / v_{0}, D_{0}=0.03$ and the compartment geometry is as in Fig. 2 Inset: $\eta$ vs. $\tau_{\theta}$ for large $\Omega$, see main panel (b).

readily extended to more realistic Janus swimmers of finite radius; (iv) On running our integration code for $\theta$ randomizing b.c. (not shown), we obtained substantially smaller $\eta$ values. This is a consequence of the fact that the persistency of the selfpropulsion velocity is suppressed by the particle collisions against the walls. This effect gets more pronounced with increasing $D_{0}$, as thermal noise causes more wall collisions and, thus, stronger $\theta$ randomization at the boundaries 4 .

Chiral Janus particles, $\Omega>0$. An angular bias $\Omega$ affects the autonomous ratchet effect discussed so far only in the strong chirality regime. In Fig. 5 we present numerical simulation results for levogyre JP's, $\Omega>0$, diffusing in the triangular channel of Fig. 2. As expected, on increasing $\tau_{\theta}$ the rectification power approaches an horizontal asymptote 35, see Fig. 5(a). However, such an asymptote gets lower at higher $\Omega$, until the curves $\eta=\bar{v} / v_{0}$ and, therefore, $\bar{v}$ versus $\tau_{\theta}$ develop a distinct maximum, see insets of panels (a) and (b). This change marks the crossover between the regimes of weak and strong chirality. Indeed, the chiral nature of the JP dynamics can be fully appreciated when the autocorrelation time of its self-propulsion velocity, $\tau_{\theta} / 2$, is of the order of the reciprocal of the cyclotron frequency, $\Omega$, namely for $\tau_{\theta} \simeq 2 / \Omega$. This simple argument closely locates the maxima of $\bar{v}$ in both insets of Fig. 5 .

The dependence of the rectification power on $\Omega$ is illustrated in panel (b) of Fig. 5. Independently of the level of translational noise, $D_{0}, \eta$ is largely insensitive to $\Omega$ until to a certain value, after which it suddenly drops to zero. Such a threshold value, termed here $\Omega_{M}$, can be estimated by noticing that on increasing $\Omega$ the chiral radius $R_{\Omega}=v_{0} /|\Omega|$ decreases, until the microswimmer can perform a full circular orbit inside the compartment, without touching the channel walls (actually a logarithmic spiral with exponentially small steps [18). In the noiseless limit, this happens for $2 R_{\Omega} \simeq x_{L}$, that is, $\Omega_{M} \simeq 2 v_{0} / x_{L}$. Of course, this argument holds under the additional condition that $\Omega_{M} \tau_{\theta}>1$, to ensure a sufficiently long self-propulsion time. This estimate of $\Omega_{M}$ is in close agreement with the data of Fig. 5(b) and will be used to explain the peaks developed by $\eta$ in Fig. 7(b) of Sec. 3.2

Another remarkable result of this section is reported in the inset of Fig. F(b): At very high $\Omega$, the horizontal asymptote of $\bar{v}$ changes sign. This is an instance of the current reversal phenomenon one often encounters in the ratchet literature 2. When the chiral radius grows so large that the chirality of the JP does not affect much its pore crossing, $R_{\Omega} \gtrsim \Delta$, self-propulsion generates an effective translational (colored) noise, with time constant $\tau_{\theta} / 2$, which adds to the white noise of constant $D_{0}$, as detailed in Secs. 4.1 and 4.2 . Under these circumstances, Eq. (1) thus describes a 
thermal ratchet. On the contrary, the rectification mechanism of non-chiral particles described above is rather reminiscent of a rocked ratchet (this ratchet classification is reviewed in Ref. 2]). For a given left-right asymmetric ratchet potential, rocked and thermal ratchets tend to generate opposite rectification currents. For this reason the current reversals depicted in the inset of Fig. 5 is not totally unexpected. However, the magnitude of the currents involved is probably too small to be of practical use.

\subsection{Upside-down asymmetric channels}

The model Langevin equations (1) have been integrated in Ref. 36 to study the net flow of a levogyre microswimmer with $\Omega>0$, confined to the periodic channel of boundaries,

$$
\begin{aligned}
& w_{+}(x)=\frac{1}{2}\left[\Delta+\epsilon\left(y_{L}-\Delta\right) \sin ^{2}\left(\frac{\pi}{x_{L}} x+\frac{\phi}{2}\right)\right], \\
& w_{-}(x)=-\frac{1}{2}\left[\Delta+\left(y_{L}-\Delta\right) \sin ^{2}\left(\frac{\pi}{x_{L}} x\right)\right],
\end{aligned}
$$

where $x_{L}$ quantifies the compartment length, $\Delta$ the pore size, and $y_{L}$ the channel width. Two additional tunable geometrical parameters have been introduced in $w_{+}(x)$, namely, $\phi$ and $\epsilon$ with $\epsilon \geq 0$, respectively, to shift the position and tune the amplitude of the upper wall with respect to the lower one (a few examples of the corresponding channel compartments are drawn in Fig. 8).

When confined to a channel compartment of size smaller than its self-propulsion length, $l_{\theta}>x_{L}$, a chiral microswimmer tends to align its velocity parallel to the walls 1322 , thus generating two boundary flows oriented to opposite directions, see Fig. 1 (b). For $\Omega>0$, the JP is levogyre, which means that the upper and lower boundary flows are oriented, respectively, to the left and right. The net flow along the channel axis, $\bar{v}$, takes the sign of the flow along the least corrugated boundary, that is $w_{+}(x)$

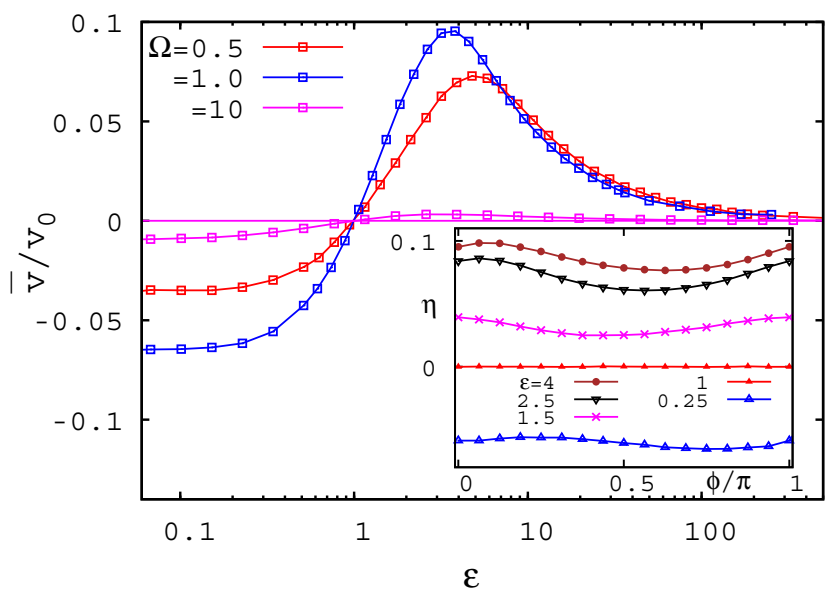

Fig. 6. (Color online) Rectification of a levogyre JP in the channel of Eq. $(3): \bar{v} / v_{0}$ vs. $\epsilon$ for $\Delta=0.12, D_{0}=0.01$ and different $\Omega$. We remind that for a right-left symmetric channel, $\bar{v}(-\Omega)=-\bar{v}(\Omega)$. Other simulation parameters are: $D_{\theta}=0.3, v_{0}=1$, and $x_{L}=y_{L}=1$. Inset: $\eta$ vs. $\phi$ for $\Omega=1$ and $\epsilon$ as in the legend. All other simulation parameters are as in the main panel. 

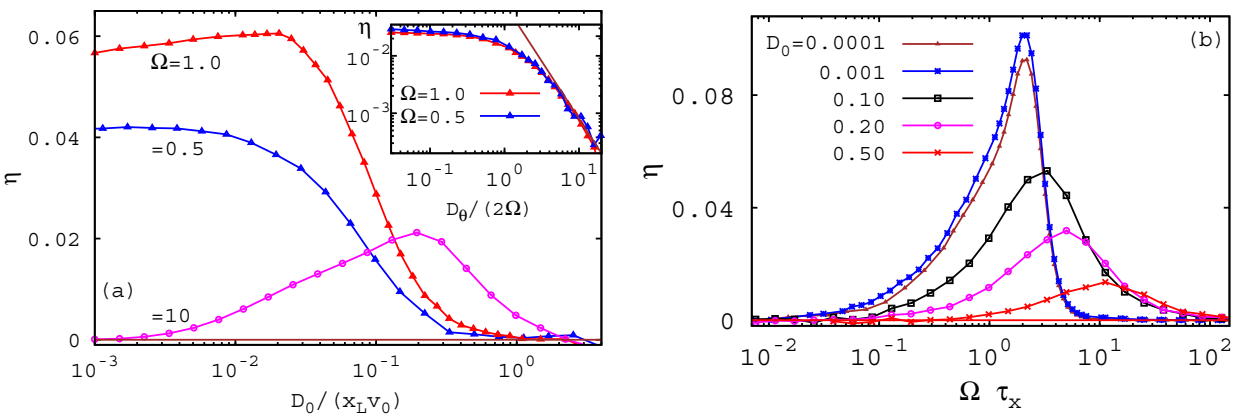

Fig. 7. (Color online) Optimization of the rectification of a levogyre JP with $v_{0}=1$ in the channel of Eq. (3) with $\epsilon=0.25, \phi=0$, and $x_{L}=y_{L}=1$ : (a) role of noise: $\eta$ vs. $D_{0}$ for $\Delta=0.08, D_{\theta}=0.1$, and different $\Omega$ (see legends). Inset: $\eta$ vs. $D_{\theta}$ for $D_{0}=0.05, \Delta=0.08$ and different $\Omega$; (b) role of frequency: $\eta$ vs. $\Omega$ for $\Delta=0.12, \tau_{\theta}=10, D_{0}=0.1, \tau_{x}=x_{L} / v_{0}$, and different $D_{0}$ (see legend).

for $\epsilon<1$ and $w_{-}(x)$ for $\epsilon>1$ and vanishes for $\epsilon=1$. This mechanism explains the current reversals shown in Fig. 6 .

The dependence of $\eta$ on the fluctuation parameters $D_{0}$ and $D_{\theta}$ is illustrated in Fig. 7] adapeted from [36, and in particular in panel (a). The rectification power is proportional to $\left(\Omega \tau_{\theta}\right)^{2}$ in the weak chirality regime, i.e., inverse proportional to $D_{\theta}^{2}$ [see inset of Fig. 7(a)]. In the opposite limit of strong chirality, $\Omega \tau_{\theta} \gg 1, \eta$ approaches a maximum, which depends on $D_{0}$, the chiral radius $R_{\Omega}$ and the compartment geometry. The curves of $|\bar{v}|$ versus $D_{0}$ increase (decrease monotonically) at high (low) frequency; they all eventually decay to zero for $D_{0} \gg v_{0}|\Omega|$, no matter what the value of $\Omega$.

For an optimal value of $\Omega$ and low noise levels, the particle tends to accumulate against the walls 37/4, with tangential velocities close to $\pm v_{0}$. This is the condition of strong chirality, $|\Omega| \tau_{\theta} \gg 1$, and low noise, $v_{0}|\Omega| / D_{0} \gg 1$, where strong autonomous rectification was first reported [36. Indeed, as the chiral radius exceeds the compartment dimensions, $R_{\Omega} \gg x_{L}$, a strongly chiral swimmer spends more time drifting between the upper and lower walls than sliding along them, thus weakening the boundary flows. On the other hand, when the chiral radius grows too short, $R_{\Omega} \ll x_{L}$, diffusion occurs mostly away from the boundaries. As a consequence, in both $R_{\Omega}$ limits the torque exerted by $\Omega$ becomes ineffective and $\bar{v}$ tends to vanish. Of course, in the weak chirality regime, $|\Omega| \tau_{\theta} \ll 1$, chirality effects are negligible, altogether.

This behavior is confirmed by the rectification peaks of the curves $\eta$ versus $\Omega$ displayed in Fig. $7(\mathrm{~b})$. On decreasing $D_{0}$ the $\eta$ peak shifts toward a limiting value, where it is the most pronounced. This is the $\Omega$ threshold value, $\Omega_{M}$, introduced in the previous Sec. 3.1. Indeed, for $\Omega \simeq \Omega_{M}$ we know already that the microswimmer can perform a circular orbit, without being captured by the boundary layers. Moreover, by closing its orbit inside a compartment, the swimmer gets trapped there, which explains the sudden drop of $\eta$ for $\Omega \geq \Omega_{M}$. The peak in the curves $\bar{v}$ versus $\epsilon$ for $\epsilon>1$ and constant $\Omega$ reported in Fig. 6 can be interpreted in the same way [36].

We have already seen that thermal noise disrupts the boundary flows by kicking the particle inside the compartment. Moreover, it also perturbs its circular orbits by making them spiral faster and their centers diffuse. That is why, on increasing $D_{0}$, the $\Omega$-peak tends to shift to higher $\Omega$ (i.e., smaller $R_{\Omega}$ ) and diminish in height, as shown in Fig. 7(b) and anticipated in Fig. 7(a). 


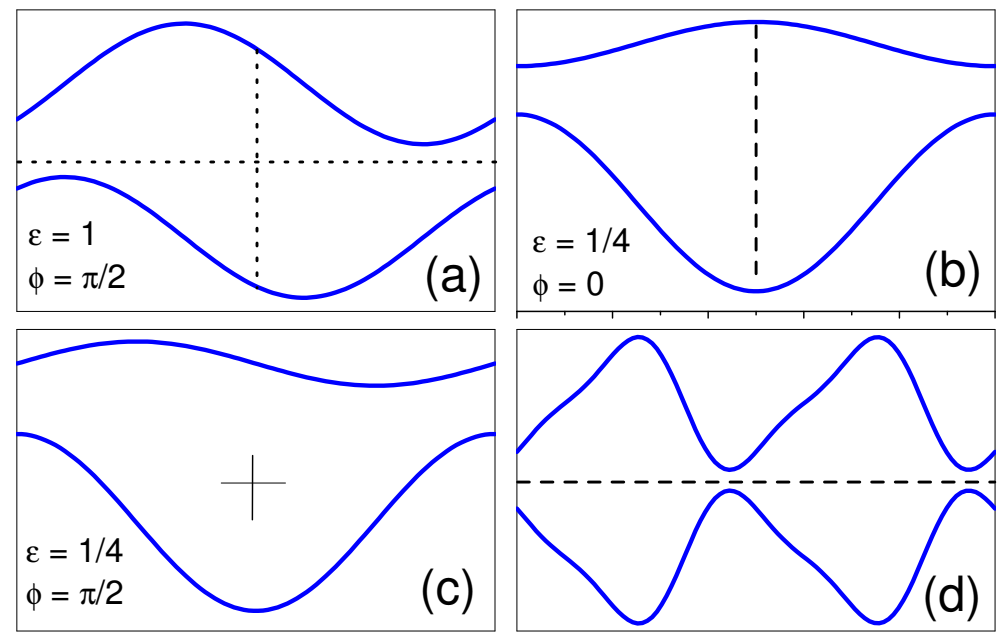

Fig. 8. (Color online) Compartments of periodically corrugated channels with different symmetry properties: (a) centro- or supersymmetric; (b) left-right symmetric; (c) asymmetric; and (d) upside-down symmetric. The channels walls, $w_{ \pm}(x)$, in (a)-(c) are given by Eqs. (3) for $\epsilon$ and $\phi$ as reported; (d) example of upside-down symmetric, left-right asymmetric channel with $w_{ \pm}(x)$ biharmonic sinusoidal functions with components of period $x_{L}$ and $x_{L} / 2$.

\subsection{Channel asymmetry requirements}

In our interpretation the rectification process is governed by the boundary flows, and therefore by the spatial symmetry of the channel walls 3 . This picture is consistent with rigorous symmetry arguments. First of all we notice that the $2 \mathrm{D}$ channel compartments in Fig. 8 can be asymmetric under inversion of either the $y$ axis $(y \rightarrow-y$, upside-down asymmetric), panel (b), of the $x$ axis $(x \rightarrow-x$, right-left asymmetric), panel (d), or both, panel (a,c). Most remarkably, compartment (a), while both upside-down and right-left asymmetric, is invariant under any pair of $x$ and $y$ axis inversions, namely, it is centro-symmetric. On combining the symmetry properties of the model dynamics, Eq. (1), with those of the channel compartment, we arrive at a few interesting conclusions:

(i) With reference to the right-left symmetric compartment (b), we notice that Eqs. (1) are invariant under the transformations $x \rightarrow-x$ and $\theta \rightarrow \pi-\theta$ or, equivalently, $\Omega \rightarrow-\Omega$, which leave the channel also invariant; hence $\bar{v}(-\Omega)=-\bar{v}(\Omega)$. As a consequence, nonchiral JP's cannot be rectified in compartment (b), as, clearly, $\bar{v}(0)=$ 0 . This last property holds in a wider sense, as discussed in item (v) below.

(ii) Analogously, for an upside-down symmetric channel one concludes that $\bar{v}(\Omega)=$ $\bar{v}(-\Omega)$. The consequence of this last symmetry relation is that for the triangular channel of Sec. $3.1 \bar{v}$ can only be a function of $\Omega^{2}$, which explains the flat branch of the $\eta$ curves with $\Omega<\Omega_{M}$, plotted in Fig. 5(b).

(iii) By shifting the channel walls $w_{ \pm}(x)$ in Eq. (3) by a length $\phi$, one can easily prove the additional symmetry relations $\bar{v}(\phi, \Omega)=\bar{v}(-\phi, \Omega)$ for right-left symmetric compartments [compare compartments (b) and (c) in Fig. 8] and $\bar{v}(\phi, \Omega)=\bar{v}(-\phi,-\Omega)$ for upside-down symmetric compartments. As displayed in the inset of Fig. 6 , $\bar{v}$ is weakly modulated by a relative shift of the walls, $\phi$, and so are the boundary flows.

(iv) For a centro-symmetric compartment, (a), both parity relations hold simultaneously; hence, $\bar{v}(\Omega)=0$. Numerical simulations for the channel of Eq. (3) with $\epsilon=1$ and any $\phi$ support this conclusion. 
(v) From items (i) and (ii) one is led to conclude that $\Omega \neq 0$ is a necessary condition for JP rectification in the right-left symmetric channels but not in the upside-down symmetric ones. We observed that this condition applies, in fact, to a wider class of compartments, which includes compartments (a)-(c) of Fig. 8. For the sake of an argument, we assume first that self-propulsion is switched off, i.e., $v_{0}=0$. As apparent from Fick-Jacobs reduction technique [138 3940], diffusion along a smooth directed channel depends on the modulating function $\sigma(x)=w_{+}(x)-$ $w_{-}(x)$. If $w_{ \pm}(x)$ are sinusoidal functions of period $x_{L}$, so is their difference, $\sigma(x)$. Accordingly, the reduced longitudinal particle dynamics would be mirror symmetric. As self-propulsion is switched on, the diffusing particle is subject to an additional time-correlated noise, which does break the time symmetry. However, due to the mirror symmetry of the reduced particle dynamics along the channel axis, this does not suffice to ensure rectification of a JP with $\Omega=0$. Put differently, a breach of the right-left symmetry of the channel compartment, does not suffice to rectify nonchiral JP's.

\section{Diffusion of active microswimmers}

As a measure of the efficiency of the autonomous rectification mechanism we now analyze the dispersion of a JP along the channel axis [41. This is an important issue experimentalists address when trying to demonstrate rectification: Indeed, drift currents, no matter how weak, can be detected over an affordable observation time only if the relevant dispersion is sufficiently small. To this purpose we compute the transport diffusivity of a JP in a channel defined as

$$
D_{\mathrm{ch}}=\lim _{t \rightarrow \infty}\left[\left\langle x^{2}(t)\right\rangle-\langle x(t)\rangle^{2}\right] /(2 t) .
$$

\subsection{Diffusion of nonchiral microswimmers, $\Omega=0$}

A full analytical investigation of the model of Eq. (1) is out of question even in the bulk and for nonchiral JP's, $\Omega=0$. However, on noticing that 442 .

$$
\langle\cos \theta(t) \cos \theta(0)\rangle=\langle\sin \theta(t) \sin \theta(0)\rangle=(1 / 2) e^{-|t| D_{\theta}},
$$

it follows immediately that the self-propulsion velocity components $v_{0, x}=v_{0} \cos \theta$ and $v_{0, y}=v_{0} \sin \theta$ can be regarded as the components of a $2 \mathrm{D}$ non-Gaussian noise $\xi_{s}(t)$ with zero mean, $\left\langle\xi_{s, i}(t)\right\rangle=0$, and finite-time correlation functions, $\left\langle\xi_{s, i}(t) \xi_{s, j}(0)\right\rangle=$ $2\left(D_{s} / \tau_{\theta}\right) \delta_{i j} e^{-2|t| / \tau_{\theta}}$, where $D_{s}=v_{0}^{2} \tau_{\theta} / 4$ and $\tau_{\theta}=2 / D_{\theta}$. In the bulk the first two LE of Eq. (1) are statistically independent and, therefore, a nonchiral particle diffuses according to Fürth's law

$$
\left\langle\Delta \mathbf{r}(t)^{2}\right\rangle=4\left(D_{0}+v_{0}^{2} \tau_{\theta} / 4\right) t+\left(v_{0}^{2} \tau_{\theta}^{2} / 2\right)\left(e^{-2 t / \tau_{\theta}}-1\right),
$$

with $\Delta \mathbf{r}(t) \equiv \mathbf{r}(t)-\mathbf{r}(0)$. Accordingly, the approximate equality $\left\langle\Delta \mathbf{r}(t)^{2}\right\rangle=4 D t$, holding for $t \gg \tau_{\theta}$, defines the particle bulk diffusivity,

$$
D=D_{0}+D_{s} \equiv D_{0}+v_{0}^{2} \tau_{\theta} / 4
$$

Of course, if the JP diffuses in a non-corrugated channel, say, with $w_{+}(x)=w_{-}(x)=$ $y_{L} / 2$, then $D_{\mathrm{ch}}=D$, as confirmed by the simulation data of Fig. 9 (dashed curves).

When confined to a sinusoidal channel, the particle diffusivity is suppressed by the geometric constrictions represented by the pores, see Figs. 9 for nonchiral and 10 


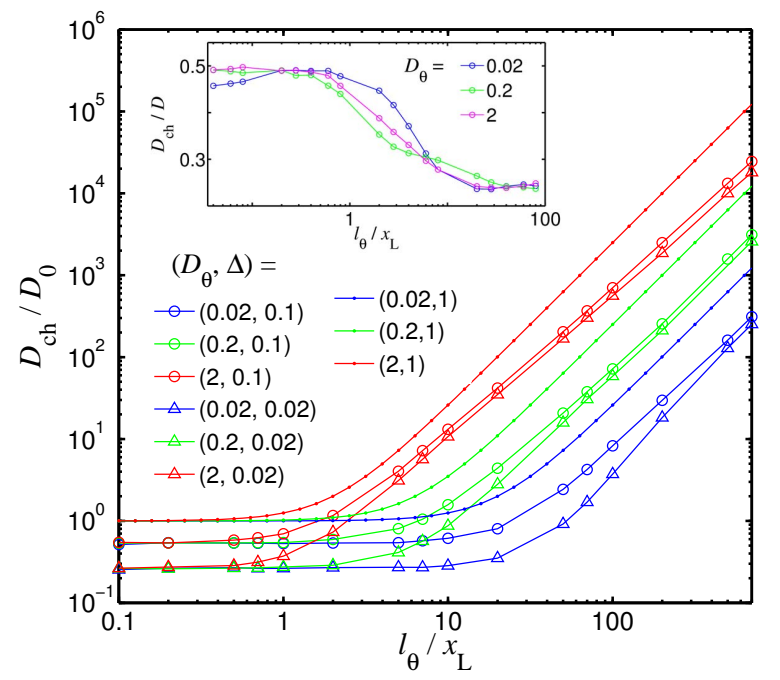

Fig. 9. (Color online) Diffusion of a levogyre JP in a fully symmetric sinusoidal channel with $w_{ \pm}(x)$ given in Eq. (3): $D_{\text {ch }}$ vs. $l_{\theta}$ for different $D_{\theta}$ and $\Delta$. Note that $\Delta=1$ represents the limiting case of a straight channel of width $\Delta=y_{L}=1$ and here $D_{\text {ch }}=D$, see Eq. (6). Other simulation parameters are $x_{L}=y_{L}=1, \epsilon=1$, and $D_{0}=0.01$. Inset: $D_{\mathrm{ch}} / D$ vs $l_{\theta} / x_{L}$ for different $D_{\theta}$ (see legend) and $D$ defined in Eq. (6). All remaining simulation parameters are as in Fig. 10 with the numerical estimates $\kappa_{0}=0.48$ and $\kappa_{s}=0.23$. We checked that our data do better approach the predicted power law, $D_{\mathrm{ch}} \propto l_{\theta}^{2}$, on further increasing $l_{\theta}$.

for chiral JP's. In the absence of self-propulsion, $v_{0}=0$, the bulk diffusivity of a nonchiral JP, Eq. [6], is $D=D_{0}$ and the channel diffusivity can be written as $D_{\mathrm{ch}}=\kappa_{0} D_{0}$, with $\kappa_{0}$ a well studied function of $\Delta$ and $D_{0}$ [3]44. In the opposite limit, $v_{0} \rightarrow \infty$, the process is governed by self-diffusion, that is, $D \simeq D_{s}$ and, accordingly, $D_{\mathrm{ch}}=\kappa_{s} D_{s}$, with $\kappa_{s}$ much less sensitive to the pore constriction than $\kappa_{0}$. Both asymptotic regimes of $D_{\text {ch }}$ are illustrated in Fig. 9 for different values of $\Delta$ and $D_{\theta}$.

This picture does not depend much on the actual compartment geometry. This conclusion is corroborated by our simulations for different channel geometries. The channel diffusivity of a nonchiral JP in the triangular channel of Sec. 3.1 is plotted in the inset of Fig. 11. Here, too, in the limit of short self-propagation times, $\tau_{\theta} \ll \tau_{x}$, $D$ approaches $D_{0}$, while we know that $\eta$ drops to zero, see Fig. 3(a). This regime amounts to an ordinary unbiased Brownian motion occurring in a triangular channel, for which $D_{\mathrm{ch}} / D_{0}=\kappa_{0}$, with $\kappa_{0}$ a function of the compartment geometry (for the compartment of Fig. 2. $\kappa_{s} \simeq 0.55$ (45)). In the opposite limit, $l_{\theta} \gg x_{L}$, as $\eta$ approaches its horizontal asymptote of Fig. 3 (a), $D$ grows like $D_{s}$, but the ratio $D_{\text {ch }} / D$ decreases toward a $\left(D_{0}\right.$-dependent) lower bound, $\kappa_{s} \simeq 0.16$.

We give next a simple phenomenological argument to compare the large- $l_{\theta}$ behaviors of $\eta$ and $D_{\mathrm{ch}} / D_{0}$ in the triangular channel of Fig. 2. The extension of this argument to the case of the sinusoidal channel considered in Figs. 9 and 10 is given in Ref. [46]. Consistently with the estimate of the bulk active diffusion, $D_{s}=v_{0}^{2} \tau_{\theta} / 4$, we can assume that a channeled JP self-propels itself to the right and left, alternately, with time constant $\tau_{\theta} / 2$. When confined to a channel compartment, its effective self-propulsion velocities to the right/left are, respectively, $v_{R, L}=\mu_{R, L} v_{0}$ with mobility constants $\mu_{R, L}$, which depend on the compartment geometry. In terms of the right/left mobility, the rectification power of Eq. (2) reads $\eta=\left(\mu_{R}-\mu_{L}\right) / 2$, and the corresponding channel diffusivity [47/48] $D_{\mathrm{ch}} / D_{0}=\left(\mu_{R}+\mu_{L}\right)^{2} / 2$. In the absence 


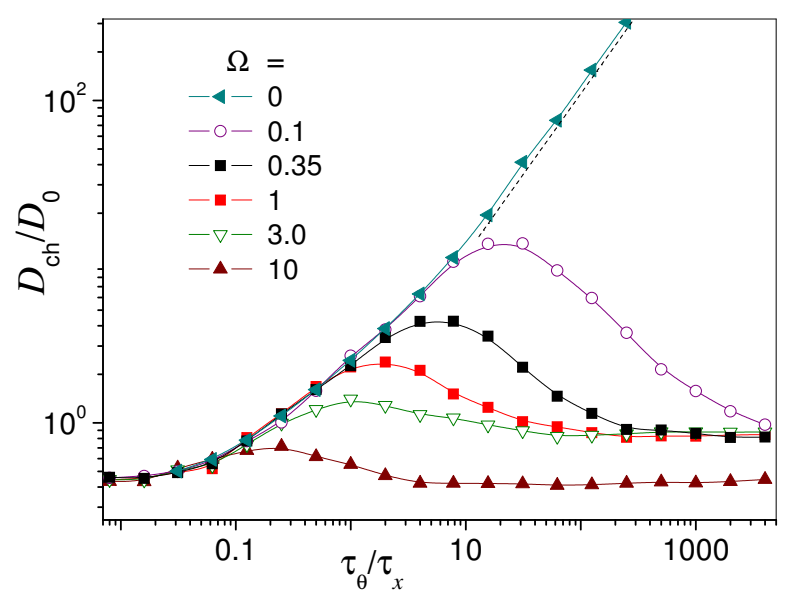

Fig. 10. (Color online) Diffusion of a levogyre JP in the sinusoidal channel of Eq. (3) $D_{\text {ch }} / D_{0}$ vs. $\tau_{\theta}$ for different $\Omega$. Here, $\tau_{x} \equiv x_{L} / v_{0}, v_{0}=1, D_{0}=0.05, \Delta=0.08$ and $\epsilon=1$. The dashed line represents the asymptotic linear power-law of Eq. 6].

of thermal noise, $D_{0}=0$, for the triangular compartment of Fig. 2 one can make use of the approximations $\mu_{L}=0$ and $\mu_{R}=\cos ^{2} \alpha / \sqrt{2}$ [46]. The ensuing estimates for $\eta$ and $D_{\mathrm{ch}}$ at zero noise, $\eta^{(0)}=0.28$ and $\kappa_{s}=D_{\mathrm{ch}} / D_{0}=0.16$, reproduce fairly closely the relevant asymptotes of Figs. 3 and 11. It should be remarked that, as discussed in Sec. 3.1, for $D_{0}>0$ thermal noise tends to suppress $\eta, \eta<\eta^{(0)}$, but increase $D_{\text {ch }}$, $D_{\mathrm{ch}}>D_{\mathrm{ch}}^{(0)}$.

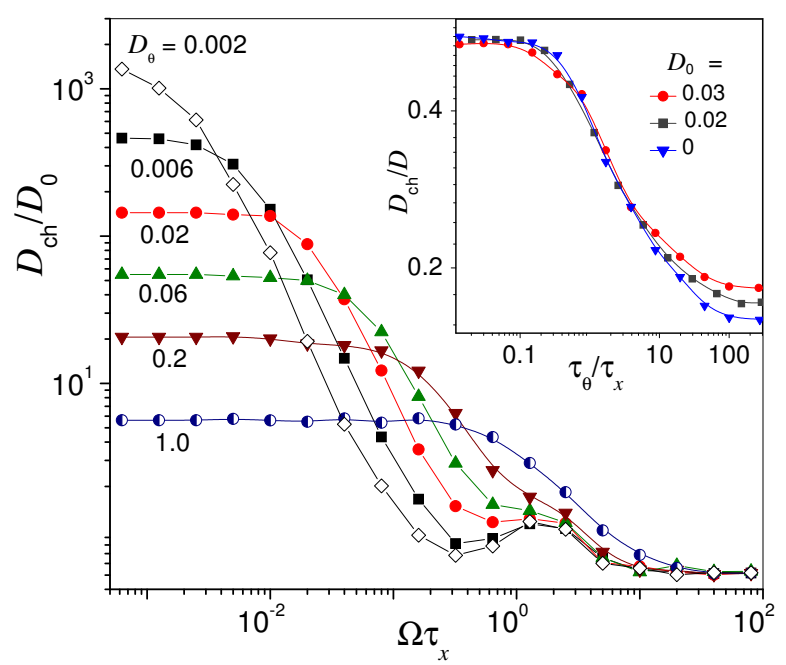

Fig. 11. (Color online) Diffusion of a levogyre JP in the triangular channel of Fig. $2 . D_{\mathrm{ch}} / D_{0}$ vs. $\Omega$ for different $D_{\theta}$. Here, $\tau_{x} \equiv x_{L} / v_{0}, D_{0}=0.03$ and the remaining simulation parameters are as in Fig. 2. The diffusion of a nonchiral JP, $\Omega=0$, is shown in the inset for different $D_{0}: D_{\text {ch }} / D$ vs. $\tau_{\theta}$ with $D$ defined in Eq. 66. 


\subsection{Diffusion of chiral microswimmers, $\Omega \neq 0$}

The transport diffusivity of chiral JP's is illustrated in Figs. 10 and 11. The dependence of $D_{\text {ch }}$ on $\Omega$ well summarizes the different chiral regimes discussed in Sec. 3 . We pointed out that chiral effects are observable only if the self-propulsion time constant $\tau_{\theta}$ is long enough, that is $|\Omega| \tau_{\theta} \gg 1$ or $R_{\Omega} \ll l_{\theta}$. On the other hand, when the chiral radius $R_{\Omega}$ grows smaller than the compartment dimensions, $R_{\Omega} \ll x_{L}$, or $|\Omega| \tau_{x} \gg 1$, chirality suppresses active transport. In general, chiral self-propulsion effects are appreciable for $\tau_{\theta} \gg \tau_{x}$, 4 .

In addition, we remark here that the $\Omega$-dependence of the bulk diffusivity of a chiral particle can be obtained from Eq. (5) by applying to our model the approach of Refs. 4950, namely

$$
D(\Omega)=D_{0}+\frac{v_{0}^{2} / \tau_{\theta}}{\left(2 / \tau_{\theta}\right)^{2}+\Omega^{2}},
$$

where $D(0)$ coincides with $D$ in Eq. (6) 46.

Similarly to the case of the nonchiral JP's discussed in the previous section, the confining action of the channel corrugations tends to suppress the particle diffusivity in the channel, $D_{\mathrm{ch}}(\Omega)$. Here too the symmetry of the walls plays no major role. For this reason we analyze the effects of chirality on active diffusion by discussing simulation data for a sinusoidal (Fig. 10) and triangular channel (Fig. 11) on the same foot.

With these premises the main features of the curves $D_{\mathrm{ch}}$ versus $\tau_{\theta}$ in a sinusoidal channel at constant $\Omega$, Fig. 10, are readily explained: (i) The curve $\Omega=0$ reproduces the situation of Fig. 9 with $D_{\text {ch }}$ growing linearly with $\tau_{\theta}$; (ii) For $D_{0} \ll D_{s}$ the channel diffusivity is proportional to $D(\Omega)$, that is $D_{\mathrm{ch}}(\Omega)=\kappa_{s} D(\Omega)$, like for the nonchiral JP's; (iii) Moreover, the corresponding maxima occur for $|\Omega| \tau_{\theta}=2$, see Eq. (7), with $D_{\text {ch }}^{\max }=D\left(2 / \tau_{\theta}\right) \simeq \kappa_{s} D_{s} / 2$; (iv) For finite $\Omega$ the activated diffusivity in the channel is suppressed both for $\tau_{\theta} \rightarrow 0$ and $\tau_{\theta} \rightarrow \infty$. Accordingly, $D_{\operatorname{ch}}(\Omega) \rightarrow \kappa_{0} D_{0}$, where $\kappa_{0}$ has been defined in Sec. 4.1.

The $\Omega$ dependence of $D_{\mathrm{ch}}$ in the triangular channel of Fig. 2 at constant $\tau_{\theta}$ is also consistent with the above interpretation of chirality effects on channel diffusivity. In addition, the data sets of Fig. 11 show that: (v) In the regime of weak chirality, $|\Omega| \tau_{\theta} \ll 1$, the $\Omega$ dependence of $D_{\text {ch }}$ is negligible, as it was for $\eta$ in Fig. 5 (b). $D_{\text {ch }}$ starts decreasing appreciably only for $|\Omega| \tau_{\theta} \gtrsim 2$, in coincidence with the $\eta$ maxima displayed in the insets of Fig. 5. (vi) In the regime of strong chirality, $|\Omega| \tau_{\theta} \gg 1$, $D(\Omega) \rightarrow D_{0}$, so that $D_{\text {ch }} \simeq \kappa D_{0}$ with $\kappa \simeq 0.55$ (see discussion of Fig. 9); (vii) Finally, small diffusivity peaks emerge also for $|\Omega| \tau_{\theta} \gg 1$. They are centered around $\Omega_{M}$ and correspond to the sudden drop in the rectification power [Fig. 5(b), main panel] that occurs when $R_{\Omega}$ grows shorter than $x_{L}$.

\section{Conclusions}

We numerically simulated the transport of artificial active microswimmers diffusing along a narrow periodically corrugated channel. Key transport quantifiers, like rectification power and diffusivity, strongly depend on the particle self-propulsion mechanism and the channel compartment geometry. Applications of such control technique are within the reach of today's technology. Specialized microfluidic circuits can be designed, for instance, to guide chiral microswimmers to a designated target. The same technique can be utilized to fabricate monodisperse chiral microswimmers (presently a challenging technological task). By the same token, microswimmers capable of inverting chirality upon binding to a load, can operate as chiral shuttles along a suitably corrugated channel even in the absence of gradients of any kind. 
The model analyzed here should be regarded as a stepping stone for more challenging generalizations and sophisticated comparisons with ongoing experimental work. Among the issues one should address next we mention: (i) diffusion gradients. Either the channel profile or the local inhomogeneities responsible for self-propulsion can be graded so as to generate an $x$-dependent channel transport diffusion coefficient, $D_{\mathrm{ch}}(x)$, which adds to the ratchet effect discussed in Sec. 3 . (ii) hydrodynamic effects. We ignored the role of the suspension fluid flowing around the moving microswimmer. An accurate account of microfluidic effects is likely to selectively impact the particle boundary flows along a corrugated channel wall as well as the translocation of finite size JP's through a narrow pore; (iii) wall interactions. The sliding b.c. implemented in our simulation code are known to reproduce rather closely certain experimental conditions, but surely are not granted in all setups under investigation. Particle translocation through narrow constrictions may be extremely sensitive to the particle-wall interactions, which thus affect both active rectification and diffusion in corrugated channels.

\section{Acknowledgements}

X.A. has been supported by the grant Equal Opportunity for Women in Research and Teaching of the Augsburg University. P.H. and G.S. acknowledge support from the cluster of excellence Nanosystems Initiative Munich (NIM). Y.L. was supported by the NSF China under grants No. 11347216 and 11334007, and by Tongji University under grant No. 2013KJ025. F.M. thanks the Alexander von Humboldt Stiftung for a Research Award. All authors thank Riken's RICC for computational resources.

\section{References}

1. For a review see: P. S. Burada, P. Hänggi, F. Marchesoni, G. Schmid, and P. Talkner, ChemPhysChem 10, 45 (2009).

2. P. Hänggi and F. Marchesoni, Rev. Mod. Phys. 81, 387 (2009).

3. S. Denisov, S. Flach, and P. Hänggi, Phys. Rep. 538, 77 (2014).

4. P. K. Ghosh, V. R. Misko, F. Marchesoni, and F. Nori, Phys. Rev. Lett. 110, 268301 (2013).

5. E. M. Purcell, Am. J. Phys. 45, 3 (1977).

6. F. Schweitzer, Brownian Agents and Active Particles (Springer, Bwrlin, 2003).

7. (a) S. Ramaswamy, Annu. Rev. Condens. Matter Phys. 1, 323 (2010); (b) T. Vicsek and A. Zafeiris, Phys. Rep. 517, 71 (2012).

8. P. Romanczuk, M. Bär, W. Ebeling, B. Lindner, and L. Schimansky-Geier, Eur. Phys. J. Special Topics 202, 1 (2012).

9. S. Jiang and S. Granick (Eds.), Janus Particle Synthesis, Self-Assembly and Applications (RSC Publishing, Cambridge, 2012).

10. A. Walther and A. H. E. Müller, Chem. Rev. 113, 5194 (2013).

11. W. F. Paxton, S. Sundararajan, T. E. Mallouk, and A. Sen, Angew. Chem. Int. Ed. 45, 5420 (2006).

12. J. G. Gibbs, and Y.-P. and Zhao, Appl. Phys. Lett. 94, 163104 (2009); J. R. Howse, R. A. L. Jones, A. J. Ryan, T. Gough, R. Vafabakhsh, R. Golestanian, Phys. Rev. Lett. 99, 048102 (2007).

13. G. Volpe, I. Buttinoni, D. Vogt, H.-J. Kümmerer, and C. Bechinger, Soft Matter 7, 8810 (2011).

14. H. R. Jiang, N. Yoshinaga, and M. Sano, Phys. Rev. Lett. 105, 268302 (2010).

15. L. Baraban, R. Streubel, D. Makarov, L. Han, D. Karnaushenko, O. G. Schmidt, and G. Cuniberti, ACS Nano 7, 1360 (2013). 
16. see, e.g., Y. Hong, D. Velegol, N. Chaturvedi, and A. Sen, Phys. Chem. Chem. Phys. 12, 1823 (2010).

17. A. Búzás, L. Kelemen, A. Mathesz, L. Oroszi, G. Vizsnyiczai, T. Vicsek, and P. Ormos, Appl. Phys. Lett. 101, 041111 (2012).

18. S. van Teeffelen and H. Löwen, Phys. Rev. E 78, 020101 (2008).

19. B. M. Friedrich and F. Jülicher, Phys. Rev. Lett. 103, 068102 (2009).

20. C. J. Brokaw, J. Exp. Biol. 35, 197 (1958); J. Cell. Comp. Physiol. 54, 95 (1959).

21. M. Mijalkov and G. Volpe, Soft Matter 9, 6376 (2013).

22. F. Kümmel, B. ten Hagen, R. Wittkowski, I. Buttinoni, R. Eichhorn, G. Volpe, H. Löwen, and C. Bechinger, Phys. Rev. Lett. 110, 198302 (2013).

23. A. Boymelgreen, G. Yossifon, S. Park, and T. Miloh, Phys. Rev. E 89, 011003(R) (2014). 24. A. Sen, M. Ibele, Y. Hong, and D. Velegol, Faraday Discuss. 143, 15 (2009).

25. A. Zöttl and H. Stark, Phys. Rev. Lett. 108, 218104 (2012).

26. P. K. Radtke and L. Schimansky-Geier, Phys. Rev. E, 85, 051110(R) (2012).

27. T. R. Kline, W. F. Paxton, T. E. Mallouk, and A. Sen, Angew. Chem. Int. Ed. 44, 744 (2005).

28. B. ten Hagen, S. van Teeffelen and H. Löwen, J. Phys.: Condens. Matter 23, 194119 (2011).

29. S. E. Spagnolie and E. Lauga, J. Fluid Mech. 700, 105 (2012).

30. M. Ripoll, P. Holmqvist, R. G. Winkler, G. Gompper, J. K. G. Dhont, and M. P. Lettinga, Phys. Rev. Lett. 101, 168302 (2008).

31. I. Buttinoni, J. Bialkè, F. Kümmel, H. Löwen, C. Bechinger, and T. Speck, Phys. Rev. Lett. 110, 238301 (2013)

32. D. Takagi, J. Palacci, A. B. Braunschweig, M. J. Shelley, and J. Zhang, Soft Matter, 10, $1784(2014)$.

33. P. K. Ghosh, P. Hänggi, F. Marchesoni, F. Nori, and G. Schmid, Europhys. Lett. 98, 50002 (2012); Phys. Rev. E 86, 021112 (2012).

34. H. Brenner and D. A. Edwards, Macrotransport Processes (Butterworth-Heinemann, New York, 1993)

35. P. K. Ghosh, P. Hänggi, F. Marchesoni, and F. Nori, Phys. Rev. E 89, 062115 (2014)

36. Y. Li, P. K. Ghosh, F. Marchesoni and B. Li, submitted (2014).

37. Y. Fily, A. Baskaran, and M. F. Hagan, arXiv:1402.5583 [cond-mat.soft].

38. A. Fick, Ann. Phys. Chem. 94, 59 (1855).

39. M. H. Jacobs, Diffusion processes (Springer, New York, 1967).

40. R. Zwanzig, J. Phys. Chem. 96, 3926 (1992).

41. L. Machura, M. Kostur, P. Talkner, J. Luczka, F. Marchesoni, and P. Hänggi, Phys. Rev. E 70, 061105 (2004).

42. Y. Fily and M. C. Marchetti, Phys. Rev. Lett. 108, 235702 (2012).

43. P. S. Burada, G. Schmid, D. Reguera, J. M. Rubi, and P. Hänggi, Phys. Rev. E 75, 051111 (2007).

44. L. Bosi, P. K. Ghosh, and F. Marchesoni, J. Chem. Phys. 137, 174110 (2012).

45. F. Marchesoni and S. Savel'ev, Phys. Rev. E 80, 011120 (2009).

46. details are given in the $\mathrm{PhD}$ thesis of Xue Ao (Augsburg University, in preparation).

47. M. Borromeo and F. Marchesoni, Chem. Phys. 375, 536 (2010).

48. M. Borromeo, F. Marchesoni, and P. K. Ghosh, J. Chem. Phys 134, 051101 (2011).

49. J. B. Taylor, Phys. Rev. Lett. 6, 262 (1961).

50. B. Kurşunoğlu, Phys. Rev. 132, 21 (1963). 